\title{
MANAGEMENT OF IATROGENIC FURCATION PERFORATION IN MANDIBULAR FIRST MOLAR WITH BIODENTINE- TWO YEARS FOLLOW UP
} Savita Thakur ${ }^{1}$, Damanpreet ${ }^{2}$, Anuradha Rani ${ }^{3}$, Nisha Garg ${ }^{4}$

${ }^{1}$ Sr. Lecturer Department of Conservative Dentistry and Endodontics, Bhojia Dental College and Hospital, Himachal Pradesh, India

${ }^{2}$ Reader, Department of Conservative Dentistry and Endodontics, Bhojia Dental College and Hospital, Himachal Pradesh, India

${ }^{3}$ Professor \& Head, Dept. of Conservative Dentistry and Endodontics, HIDS, Himachal Pradesh, India

${ }^{4}$ Professor \& Head, Dept. of Conservative Dentistry and Endodontics, HIDS, Himachal Pradesh, India

\begin{abstract}
Successful management of furcation perforations poses a challenge for a clinician. The perforation can result from iatrogenic causes, caries or resorption. It is advisable to repair the perforation as soon as it is identified, since any delay allows the bacterial ingress leading to a complicated endodontic-periodontal lesion and ultimately in the treatment failure. This case report describes successful management of a furcal perforation in a mandibular first molar, which was repaired using Biodentine. The endodontic treatment was completed and the tooth was coronally restored with composite followed by full coverage restoration. Two years follow up of the case showing absence of pain; periradicular healing of the lesion, along with functional tooth stability indicated a successful outcome of endodontic treatment and sealing the perforation using Biodentine.
\end{abstract}

Key words: Biodentine, perforation, mandibular first molar.

\section{INTRODUCTION}

In endodontic practice, procedural accidents are frequently encountered that may adversely affect the prognosis of the root canal treatment. One of the common procedural accidents is an endodontic perforation.

An endodontic perforation may be defined as an artificial opening in a tooth or its root, created by boring, piercing, cutting or pathologic resorption, which results in a communication between the pulp space and the periodontal tissues. ${ }^{2}$ A furcation perforation is one such complication that refers to mid-curvature opening into the periodontal ligament (PDL) space and leads to worst possible treatment outcome.

Corresponding Author: Savita Thakur

E-mail:

thakursavita72@yahoo.in

Received: $20^{\text {th }}$ January 2017

Accepted: $3^{\text {rd }}$ March 2017

Online: $20^{\text {th }}$ May 2017
Due to the perforation bacterial infection can occur which leads to gingival growth of epithelium into the perforation area, inflammation, bone resorption and/or necrosis, eventually leading to treatment failure. Perforation can be managed either surgically or nonsurgically and the prognosis is generally excellent if the problem is well diagnosed and the repair is performed without any delay with a material which can provide proper sealing ability and biocompatibility. ${ }^{3}$

Historically, different materials have been used for furcal perforations, including amalgam, Intermediate Restorative Material (IRM), SuperEBA, Cavit, Glass Ionomer, Composites and MTA. Although, above listed materials are reasonably biocompatible and result in successful repair of the perforation, however concomitant regeneration of the lost periodontium is the elusive goal of the present day dentistry. Biodentine is a comparatively newer bioactive calcium silicate based material for crown and root dentin repair, repair of perforations, apexifications, resorption repair and rootend fillings. 
This case report describes the repair of a furcal perforation in the mandibular tooth using Biodentine, with a 24 months follow up showing absence of clinical and radiographic pathological conditions suggestive of a successful treatment outcome.

\section{CASE REPORT:}

A 45-year-old woman reported with pain in relation to left lower back tooth region since 3 days. Looking into the history it was recorded that patient had undergone a procedure for root canal treatment of mandibular $1^{\text {st }}$ molar on that side around 1 week back from a local dentist. Presently the pain was continuous, aggravated on chewing food and was relieved on taking medication. Periradicular radiographic examination revealed a little radiolucent area in the furcal region of left first mandibular molar and apical radiolucencies from pulp necrosis were also observed [Figure 1]. Several treatment options were discussed with the patient and she opted for non surgical endodontic treatment of the tooth along with perforation repair.

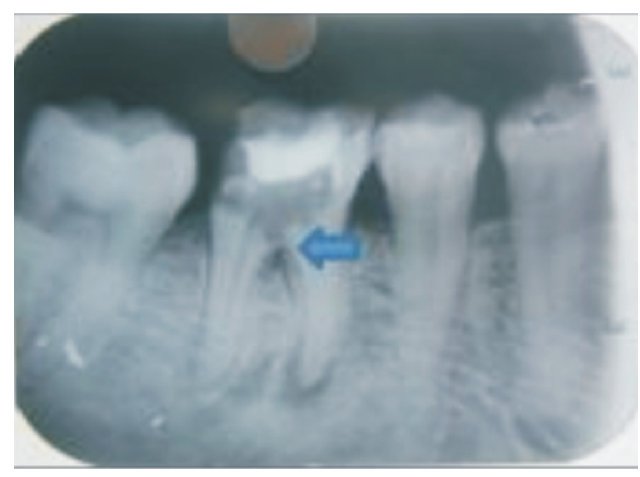

Figure 1: Pre operative IOPAR w.r.t. 36 showing furcal perforation and periapical radiolucencies

After administration of local anesthesia 2\% lidnocaine 1:100000, the tooth was isolated with a rubber dam, temporary restorative material was removed and the access cavity was prepared so that perforation area could be clinically seen (Figure 2). Hemorrhage was controlled with copious irrigation of $0.9 \%$ saline solution. A cotton pellet was placed in the orifice of perforation. The working length was then checked by using an apex locator radiovisigraphy (Figure 3). The root canals were cleaned and shaped using K- files (Dentsply) in a step back technique.

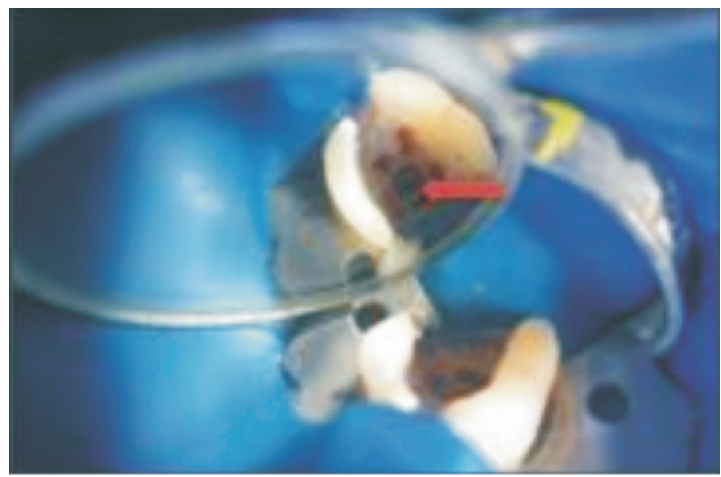

Figure 2: Clinical photograph showing modified access cavity and perforation in furcation w.r.t 36

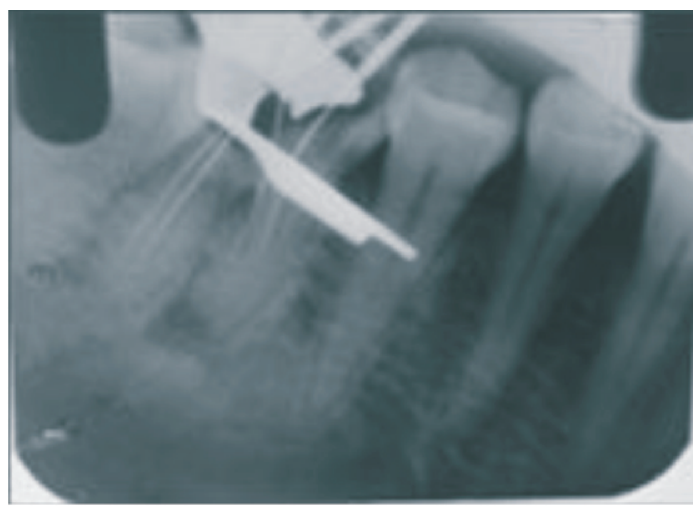

Figure 3: IOPAR w.r.t 36 (working length determination after rubber dam application)

Before the use of each instrument, irrigation of the canal was performed using a syringe $1 \mathrm{~mL}$ of $2 \%$ chlorhexidine (CHX), and immediately rinsed afterwards with $3 \mathrm{~mL}$ of saline solution. After the root canals were dried with paper points, they were obturated using lateral condensation.

After obturation of the root canals, cotton pellet was removed from the perforation, exposing the site of perforation. The furcal perforation was irrigated with saline solution and $2 \%$ Chlorhexidine, Biodentine was mixed on a paper pad, and the perforation site was repaired by condensing Biodentine in the area (Figure 4). Patient was recalled after 1 week for post endodontic composite restoration. Radiograph obtained after the restoration confirmed that perforation had been sealed. The patient was completely asymptomatic after 1 week and routinely evaluated for healing after 1 month, 6 months, 12 months and 24 months. 


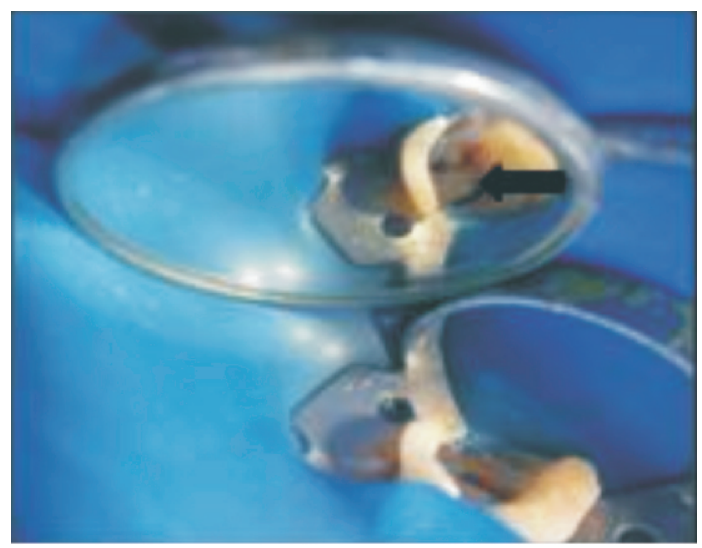

Figure 4: Perforation repair with Biodentine w.r.t.36

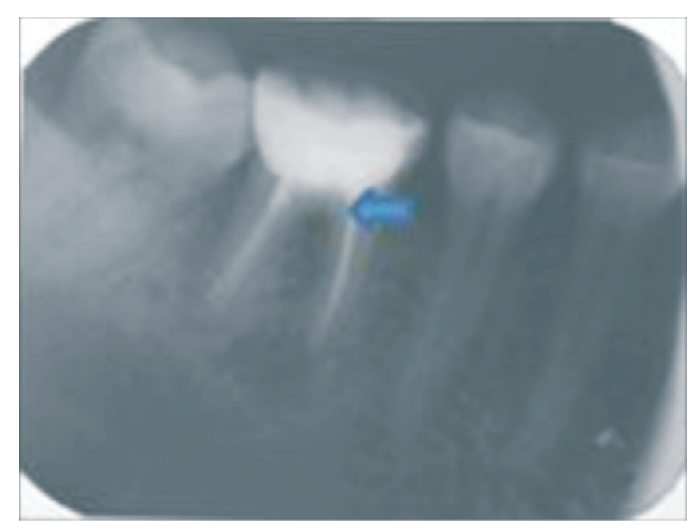

Figure 5: IOPAR at 2 years follow-up w.r.t 36

At two-year follow up, the tooth was asymptomatic and clinical examination revealed that the tooth had no pain or no tenderness on percussion and palpation. No attachment loss was seen as indicated by normal probing depths. The tooth revealed adequate clinical function, and radiographic findings showed adequate sealing of the perforation site with no radioluency finding at the furcation area and satisfactory healing of the periapical lesions.

\section{DISCUSSION}

Successful management of furcation perforations poses a challenge for a clinician. Perforations can result from iatrogenic causes, caries, or resorption. A study has reported that perforations are the second greatest cause of failures accounting for $9.62 \%$ of all unsuccessful cases. ${ }^{5}$ Therefore, this communication between the root canal system and the periodontal apparatus should be sealed with a biocompatible material as soon as possible, since any delay will allow the bacterial ingress leading to complicated endodontic-periodontal lesion. An ideal perforation repair material should provide an adequate seal, be biocompatible, dimensionally stable, insoluble, radiopaque, and allow easy manipulation and placement. ${ }^{5}$

For many decades since 1928, calcium hydroxide has been standard material for maintaining the vitality of pulp since it is capable of stimulating tertiary dentin formation. $\mathrm{Ca}(\mathrm{OH}) 2$ has been suggested as a traditional agent to manage perforations. ${ }^{6}$ However, Petersson et al. and Bogaerts stated that materials based on $\mathrm{Ca}(\mathrm{OH}) 2$ as a main ingredient were not suitable for crestal and furcation perforations because of the initial inflammatory response to these materials, which could lead to breakdown of the supporting tissues and subsequent pocket formation. ${ }^{6}$ In addition Schuurs et al. suggested that $\mathrm{Ca}(\mathrm{OH}) 2$ in contact with tissue fluids may be displaced as a result reliable seal cannot be achieved; in such situations, a more conventional restorative material such as MTA is required. ${ }^{7}$ Mineral Trioxide Aggregate introduced by Torabinejad M. in 1990 was used as a material of choice for all dentinal defects due to its biocompatibility and ability to induce calcium phosphate precipitate at interface to periodontium and bone tissue repair. However MTA has some inherent disadvantages such as long setting time - 45 mins to 2 hour, complicated handling properties and discoloration of teeth.

Therefore, quest for newer and better materials is never ending especially in the field of dental science. Various materials have been formulated, tested, and standardized to obtain maximum benefits for good clinical performance. One such new material introduced is Biodentine, which has found numerous clinical applications for repair of dentin in crown and root structure of teeth.

Biodentine ${ }^{\circledR}$ is a calcium silicate-based bioactive material introduced in 2010 by Gilles and Olivier. ${ }^{8}$ Biodentine (Septodont, Saint Maur des Fosses, France) powder is mainly composed of tricalcium silicate, calcium carbonate and zirconium oxide as the radio-pacifier and Biodentine liquid 
contains calcium chloride as the setting accelerator and water as reducing agent.

The desirable properties of biodentine make it a useful material in repairing the root and furcal perforations. Biodentine induces early mineralization by increasing the secretion of TGF- $\beta 1$ (transforming growth factor - beta 1) therefore it is also capable of angiogenesis, recruitment of progenitor cells, cell differentiation, and mineralization. ${ }^{9}$

The furcation area is subjected to masticatory forces; the repair material used will also bear the brunt of the forces during tooth function. Therefore it is necessary that the repair material have sufficient push out strength to counter these forces. Biodentine has a high compressive strength of $220 \mathrm{MPa}$ and elastic modulus of $22 \mathrm{GPa}$, which is very similar to dentin. Vivek et al 2013 has shown that MTA had significantly less push-out bond strength than Biodentine even the blood contamination had no effect on the perforations repaired with Biodentine. ${ }^{5}$

Thus ease of manipulation, better consistency, biocompatibility, bioactivity, biomineralization and safe handling with favorable setting kinetics (initial setting time 6 minutes and a final setting time of 12 minutes), makes Biodentine a suitable and preferable material for the treatment of furcal perforations, with the goal of regenerating periodontal attachment.

\section{CONCLUSION}

In the present case, furcal perforation of the mandibular left first molar was treated using nonsurgical placement of Biodentine. The repaired tooth was clinically and radiographically healthy and continued to satisfy the functional demands. Based on the outcome of the present case, Biodentine can be considered as the material of choice for the repair of furcal perforations, though further studies are required to extend the future scope of this material regarding the clinical applications.

\section{REFERENCES}

1. Deeb M, Tabjbi A,Jensen J. An evaluation of the use of amalgam, cavit and calcium hydroxide in the repair of furcation perforation. J Endod 1982; 10 (8): 459- 466.

2. Wong R. Microscopic management of procedural errors. Dent Clin North Am 1997;41:455-77.

3. Silva E, Andrade C, Tay L, Herrera D. Furcal-perforation repair with mineral trioxide aggregate: Two years follow-up. Indian J Dent Res 2012;23(4)542-545.

4. Behnia A, Strasseler HE, Campbell R. Repairing iatrogenic root perforations. JAm Dent Assoc 2000;131:196-201.

5. Vivek Aggarwal, Mamta Singla, Sanjay Miglani, Sarita Kohli. Comparative evaluation of push-out bond strength of ProRoot MTA, Biodentine, and MTA Plus in furcation perforation repair. J Conserv Dent 2013;16(5):462-465.

6. Z. Mohammadi, P. M. H. Dummer. Properties and applications of calcium hydroxide in endodontics and dental traumatology. Int Endod J 2011;44:697-730.

7. Schuurs AH, Gruythuysen RJ, Wesselink PR. Pulp capping with adhesive resin-based composite vs. calcium hydroxide: a review. Endod \& Dent Traumatol 2000;16:240-50

8. Maria Giovanna Gandolfi, Francesco Siboni, Antonella Polimeni, Maurizio Bossu, Francesco Riccitiello, Sandro Rengo and Carlo Prati. In Vitro Screening of the ApatiteForming Ability, Biointeractivity and Physical Properties of a Tricalcium Silicate Material for Endodontics and Restorative Dentistry. Dent. J. 2013;1:41-60.

9. Priyalakshmi.S, Manish Ranjan. Review on Biodentine - A Bioactive Dentin Substitute. IOSR-JDMS 2014;13(1):13-17.

Source of Support: Nil, Conflict of Interest: None Declared 